Forum 2020 · 35:255

https://doi.org/10.1007/s12312-020-00781-4

Online publiziert: 3. April 2020

C) Springer Medizin Verlag $\mathrm{GmbH}$, ein Teil von

Springer Nature 2020

SACHSEN-ANHALTISCHE

KREBSGESELLSCHAFT E.V.

Sachsen-Anhaltische Krebsgesellschaft e.V.

Halle, Deutschland

\title{
Geriatrische Onkologie im Fokus der Sachsen-Anhaltischen Krebsgesellschaft
}

elf Außenberatungsstellen im gesamten Bundesland, die jeweils einmal pro Monat besetzt werden. Selbst diese sind aber bei oft schlechter Verkehrsanbindung von den Wohnorten im ländlichen Raum und fehlender Fahrtkostenübernahme für die älteren Krebsbetroffenen vielfach schwer erreichbar.

Frau Dr. Heike Schmidt, Ärztin und Leiterin der Arbeitsgruppe „Lebensqualität" an der Universitätsmedizin Halle, hat neue Versorgungsstrategien für die geriatrische Onkologie konzipiert und untersucht. In einer Studie (Patientenzentriertes Interdisziplinäres Behandlungs- und Versorgungskonzept für Onkologischgeriatrische Patienten, PIVOG) wurden 100 ältere Tumorpatienten des Universitätsklinikums Halle vor Therapiebeginn einer umfangreichen Testung in Form eines geriatrisches Assessments unterzogen, um ihre Ressourcen und Einschränkungen in die Behandlungsplanung einzubeziehen. Nach Therapieende erhielten die Patienten ein Entlassungsgespräch und eine zusätzliche telefonische Beratung durch eine onkologische Fachpflegekraft.
Weiterentwicklungen dieser Maßnahmen werden nun in Folgestudien untersucht und in die Versorgungsangebote der Universitätsmedizin Halle überführt.

Beim Deutschen Krebskongress 2020 in Berlin konnte das Team aus der Modellregion Sachsen-Anhalt seine Erfahrungen in der Sitzung „Optimale Beratung und Versorgung älterer Krebspatienten: Impulse aus Sachsen-Anhalt" unter der Moderation von Prof. Dr. Dirk Vordermark, stellvertretender Vorsitzender der SAKG und Direktor der Universitätsklinik für Strahlentherapie in Halle, mit Fachleuten aus anderen Bundesländern diskutieren. Das Fazit der SAKG lautete: „Wir haben in Sachsen-Anhalt nicht nur Probleme, sondern auch innovative Lösungsvorschläge.“

\section{Korrespondenzadresse}

\section{Prof. Dr. Dirk Vordermark}

Sachsen-Anhaltische Krebsgesellschaft e.V. Paracelsusstr. 23, 06114 Halle (Saale),

Deutschland

info@krebsgesellschaft-sachsenanhalt.de sind laut Dr. Medenwald weitere Besonderheiten des Gesundheitsverhaltens in Sachsen-Anhalt wie eine geringe Teilnahmerate an Krebsfrüherkennungsuntersuchungen oder ein stark ausgeprägter Nikotinkonsum zu beachten. Die Belastung mit kardiovaskulären Komorbiditäten ist ebenfalls hoch, was die Therapierbarkeit von Tumorerkrankungen beeinflussen dürfte.

Der Geschäftsführer der SAKG, Sven Weise, hat in der Praxis der psychosozialen Beratung erfahren, wie schwierig die Angebote der Krebsgesellschaft oder auch onkologischer Versorger für ältere Tumorpatienten zugänglich sind. Die SAKG betreibt neben den Psychosozialen Krebsberatungsstellen in Halle und Magdeburg

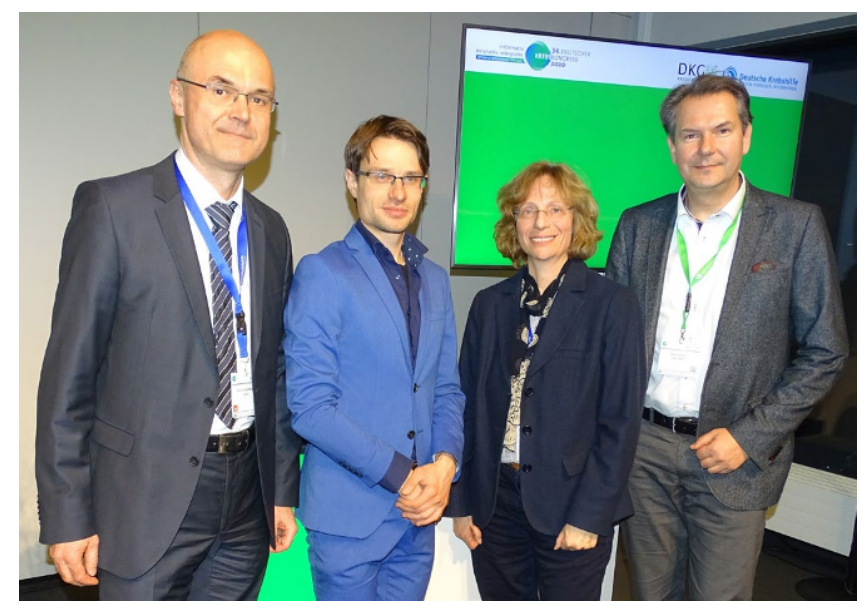

$\varangle$ Prof. Dr. Dirk Vordermark, PD Dr. Daniel Medenwald, Dr. Heike Schmidt, Sven Weise (v. I. n.r.; (c) Simone Pareigis mit freundlicher Genehmigung) 